\title{
ON THE RELATIVE GROUP COHOMOLOGY RING
}

\author{
G. R. CHAPMAN ${ }^{1}$
}

\begin{abstract}
The product structure on the Hochschild-Serre spectral sequence generalizes to the spectral sequences of ButlerHorrocks. It is shown that Evens' proof of the finite generation of the integral cohomology ring of a finite group does not generalize to the relative cohomology groups of Adamson.
\end{abstract}

Introduction. If $G$ is a finite group, $N \triangleleft G$ and $k$ is a $G$-ring, then the product on the Hochschild-Serre spectral sequence $H^{*}\left(G / N, H^{*}(N, k)\right) \Rightarrow$ $H^{*}(G, k)$ arising from the cup product on the $E_{2}$ term induces (up to sign) the cup product on $H^{*}(G, k)$ [5]. Evens [3] uses the product structure on a certain Hochschild-Serre spectral sequence to prove that $H^{*}(G, Z)$ is finitely generated, where $Z$ denotes the integers with trivial $G$-module action. Let $G_{2} \subseteq G_{1}$ be subgroups of $G$. Adamson [1] and Hochschild [4] study the class of $G_{2}$-split sequences of $G$-modules and denote the corresponding relative derived functors of $\operatorname{Hom}_{G}, \otimes_{G}$ by $\operatorname{Ext}_{\left(G, G_{2}\right)}, \operatorname{Tor}^{\left(G, G_{2}\right)}$. Then $\operatorname{Tor}_{n}^{\left(G, G_{2}\right)}(Z,-)=H_{n}\left(G, G_{2} ;-\right), \operatorname{Ext}_{\left(G, G_{2}\right)}^{n}(Z,-)=H^{n}\left(G, G_{2} ;-\right)$ which are the homology and cohomology groups of $G$ relative to $G_{2}$. Butler and Horrocks [2] construct a spectral sequence depending on $G, G_{1}, G_{2}$ with coefficients in $k$ which converges to $H^{*}\left(G, G_{2} ; k\right)$ and reduces to the Hochschild-Serre when $G_{1} \triangleleft G, G_{2}=\{e\}$. In this paper, we note that a product can be introduced on the Butler-Horrocks spectral sequence which generalizes that on the Hochschild-Serre and induces a relative cup product on $H^{*}\left(G, G_{2} ; k\right)$. The question naturally arises: Can the method of Evens be generalized to the spectral sequence of [2] to prove the finite generation of $H^{*}\left(G, G_{2} ; Z\right)$ ? In this paper, we indicate how the answer depends on the nonvanishing of a relative restriction map (i.e. the composition of the $\tau$-transformation $\left.H^{*}\left(G, G_{2} ; Z\right) \rightarrow H^{*}(G, Z)[2]\right)$ and a restriction homomorphism. To establish the nonvanishing of this map, $G$ has to be embedded in a certain semidirect product in such a way that $G_{2}$ is conveniently situated. This can only be done under favourable conditions, and we prove the following theorem which shows that under these conditions, the relative restriction is identically zero. This means that Evens' method cannot be generalized.

Received by the editors March 12, 1971 and, in revised form, August 23, 1971.

AMS 1970 subject classifications. Primary 18G25, 18G40, 18H20; Secondary $20 \mathrm{~J} 05$.

Key words and phrases. Hochschild-Serre spectral sequence, relative cohomology group, $\tau$-transformation.

1 The author thanks the referee for his advice. 
THEOREM. Let $H, K$ be subgroups of $G$ such that $H$ is cyclic, normalizes $K$ and has a generator contained in the set $[G, K]_{0} \cdot K$, where $[G, K]_{0}=$ $\left\{g k g^{-1} k^{-1} ; g \in G, k \in K\right\}$. If $M$ is an $H K$-trivial $G$-module with underlying group free, then the relative corestriction $H_{n}(H, M) \rightarrow H_{n}(G, K ; M)$ and the relative restriction $\operatorname{Ext}_{(G, K)}^{n}(M, Z) \rightarrow \operatorname{Ext}_{H}^{n}(M, Z)$ are zero $(n \neq 0)$.

1. The generalization of Evens' method. Let $G_{3}$ be a central prime order subgroup of the finite group $G$. Evens [3] notes that the HochschildSerre spectral sequence $E$ of the extension $1 \rightarrow G_{3} \rightarrow G \rightarrow G / G_{3} \rightarrow 1$ (with integer coefficients) displays a periodicity $E_{2}^{p, q} \cong E_{2}^{p, q+2}$, the isomorphism being multiplication by the generating element $\xi$ of $E_{2}^{0.2}\left(=H^{2}\left(G_{3}, Z\right)\right.$ ). The hub of Evens' proof of the finite generation of $H^{*}(G, Z)$ is that the restriction $r^{2 l}: H^{2 l}(G, Z) \rightarrow H^{2 l}\left(G_{3}, Z\right)$ is epic for some $l>0$, which means that $\xi^{l} \in E_{\infty}^{0,2 l}$. This implies that, since any element of $E_{2}^{p, q}$ can be written in the form $\xi^{i l} \cdot u$ with $u \in E_{2}^{p, q-2 i l}(q-2 i l \leqq 2 l)$, the spectral sequence terminates. This termination is essentially Evens' proof. Denote by $B$ the Butler-Horrocks spectral sequence for the groups $G, G_{2} G_{3}, G_{2}$ with integral coefficients. We note that a product can be introduced on this spectral sequence such that when $G_{2}=\{e\}$ (so that $B$ becomes $E$ ) this product is that of [5]. The details are omitted, since they are not needed in the sequel. We attempt to terminate $B$ by methods parallel to those used by Evens in terminating $E$. We make the assumption $G_{2} \triangleleft G$. This involves no loss of generality, since for $G_{2} \triangleleft G$, the isomorphism $H^{*}\left(G, G_{2} ; Z\right) \cong H^{*}\left(G / G_{2}, Z\right)$ [1] is a ring isomorphism, so it is known that $H^{*}\left(G, G_{2} ; Z\right)$ is finitely generated in this case.

Suppose we prove the relative restriction

$$
\hat{r}^{2 m}: H^{2 m}\left(G, G_{2} ; Z\right) \stackrel{\tau}{\longrightarrow} H^{2 m}(G, Z) \stackrel{r^{2 m}}{\longrightarrow} H^{2 m}\left(G_{3}, Z\right)
$$

is epic for some $m>0$. Then the same would be trivially true for $r^{2 m}$, and from [2, p. 190] we would have the following commutative exact diagrams:

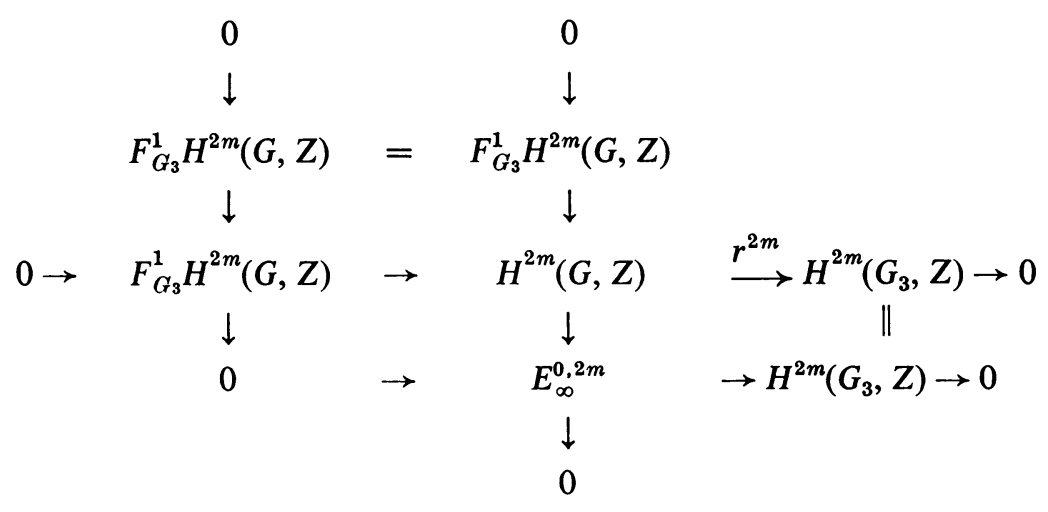




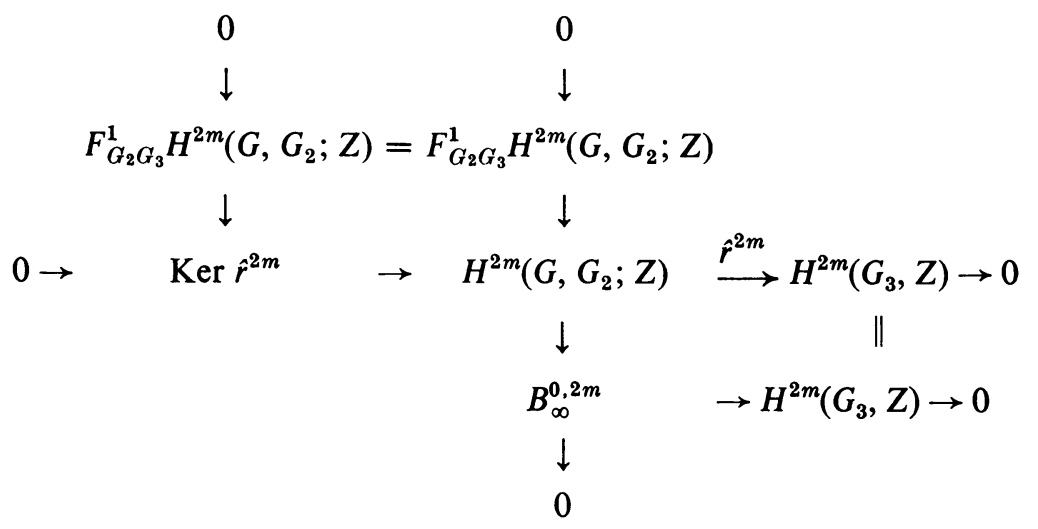

where $F_{G_{3}}^{1}, F_{G_{2} G_{3}}^{1}$ denote the subgroups consisting of those extensions which split in one place over $G_{3}, G_{2} G_{3}$ respectively. Moreover, the $\tau$ transformation $H^{2 m}\left(G, G_{2} ; Z\right) \rightarrow H^{2 m}(G, Z)$ of [2] induces a commutative map of the latter to the former, and in particular an epimorphism $B_{\infty}^{0,2 m} \rightarrow E_{\infty}^{0,2 m}$. Thus if the relative restriction were epic in some positive even degree, $\xi^{2 m}$ would have a preimage in the relative spectral sequence.

To try to obtain this result, we note that in $[3, \S 5], G$ (acting on itself by left translation) is embedded in $R$, the centralizer of $G_{3}$ in the group $S_{G}$ of permutations of $G$. Put

$$
G_{3}^{n}=G_{3} \times G_{3} \stackrel{n \text { times }}{\times} \cdots \times G_{3} .
$$

It is shown that $R$ is the semidirect product of $G_{3}^{l}$ with $S_{l}$, where $l$ is the index of $G_{3}$ in $G$, and that $H^{2 l}(R, Z) \rightarrow H^{2 l}\left(G_{3}, Z\right)$ is epic. In the relative case, $G$ acts on the set $M=\left\{g_{1} G_{2}, g_{2} G_{2}, \cdots, g_{s} G_{2}\right\}$ of left cosets of $G_{2}$ in $G$ by left translation, and if $G_{2}$ is core free this embeds $G$ in $S_{M}$, the group of permutations of $M$. If $\Gamma$ is the centralizer of $G_{3}$ in $S_{M}$, then $G \subseteq \Gamma$ and $\rho \in \Gamma$ iff $\rho h g_{i} G_{2}=h \rho g_{i} G_{2} \forall h \in G_{3}, 1 \leqq i \leqq s$. If we assume $G_{2} \cap G_{3}=\{e\}$, this means that $\rho$ is a map of $G_{2} G_{3}$ cosets, so that $\Gamma$ is the semidirect product of $G_{3}^{t}$ with $S_{t}$, where $t$ is the index of $G_{2} G_{3}$ in $G$ (see $[3, \S 5]$ ). To prove $H^{2 t}\left(\Gamma, G_{2} ; Z\right) \rightarrow H^{2 t}\left(G_{3}, Z\right)$ epic, we need a $\left(\Gamma, G_{2}\right)$-resolution. Evens' $R$-resolution is obtained by combining an $S_{l}$-resolution with a $G_{3}^{l}$-resolution. If $G_{2} \subseteq G_{3}^{t}$ it may be possible to combine a $\left(G_{3}^{t}, G_{2}\right)$ resolution with an $S_{t}$-resolution to produce a $\left(\Gamma, G_{2}\right)$-resolution. On the other hand, if $G_{2} \subseteq S_{t}$ we could try to combine a $G_{3}^{t}$ with a $\left(S_{t}, G_{2}\right)$ resolution. The latter case appears to have no meaningful interpretation. However, $G_{2} \subseteq G_{3}^{t}$ iff $\forall k \in G_{2}, \exists h_{i} \in G_{3}$ such that $k g_{i} G_{2}=h_{i} g_{i} G_{2}$ for $1 \leqq i \leqq t$. This is easily seen to be equivalent to $G_{2} G_{3} \triangleleft G$. When this is so, the image of $k \in G_{2}$ in $\Gamma$ is $h_{1} \times h_{2} \times \cdots \times h_{t}$. 
2. Proof and application of the Theorem. Among the conditions accumulated on $G_{2}, G_{3}$ are: $G_{3}$ is a central prime order subgroup of $G$, $G_{2} G_{3} \triangleleft G$ and $G_{2} \bowtie G$. The following theorem proves that under these conditions the relative restriction is identically zero in all nonzero degrees. Thus Evens' method cannot be generalized.

THEOREM. Let $H, K$ be subgroups of $G$ such that $H$ is cyclic, normalizes $K$ and has a generator contained in the set $[G, K]_{0} \cdot K$, where $[G, K]_{0}=$ $\left\{g \mathrm{~kg}^{-1} \mathrm{k}^{-1} ; g \in G, k \in K\right\}$. If $M$ is an $H K$-trivial $G$-module with underlying group free, then the relative corestriction $H_{n}(H, M) \rightarrow H_{n}(G, K ; M)$ and the relative restriction $\eta^{n}: \operatorname{Ext}_{(G, K)}^{n}(M, Z) \rightarrow \operatorname{Ext}_{H}^{n}(M, Z)$ are zero $(n \neq 0)$.

Corollary. If $G_{3}$ is a prime order central subgroup of $G, G_{2} G_{3} \triangleleft G$ and $G_{2} \bowtie G$, then $\hat{r}^{n}: H^{n}\left(G, G_{2} ; Z\right) \rightarrow H^{n}\left(G_{3}, Z\right)$ is zero $(n \neq 0)$.

Proof. Let $k \in G_{2}, g \in G$. Since $G_{2} G_{3} \triangleleft G, g k g^{-1} \in G_{2} G_{3}$-i.e. $g k g^{-1}=$ $h_{1} k_{1}$ for some $h_{1} \in G_{3}, k_{1} \in G_{2}$. Moreover, $\exists$ a choice of $k \in G_{2}, g \in G$ such that $h_{1} \neq e$, otherwise $G_{2} \triangleleft G$ contrary to hypothesis. Hence we have $h_{1}=$ $\mathrm{gkg}^{-1} k_{1}^{-1}$ for some $h_{1} \neq e \in G_{3}$. Since $G_{3}$ is of prime order, $h_{1}$ generates $G_{3}$. Thus taking $G_{2}=K, G_{3}=H$ satisfies the conditions of the theorem.

Proof of THE Theorem. Let $H=\left\{y ; y^{h}=e\right\}$ with $y \in[G, K]_{0} \cdot K$, and put $N=\sum_{i=0}^{h-1} y^{i}$. Tensors are over $Z$ unless otherwise indicated. If $W_{*} \rightarrow Z$ denotes the standard $H$-free resolution of $Z$ [6, Chapter IV], then by hypothesis $Y_{*}=\left(W_{*} \otimes M\right) \rightarrow M$ is an $H$-free resolution. Let $\beta_{*} \rightarrow M$ be the standard $(G, K)$-projective resolution of $M$ [6, Chapter IX, §8]. We prove the theorem by constructing a chain map $\psi_{*}: Y_{*} \rightarrow \beta_{*}$ extending the identity on $M$.

LEMMA. $\quad \psi_{*}$ can be chosen so that $\psi_{2 n+1}\left(Y_{2 n+1}\right) \subseteq \partial\left(\beta_{2 n+2}\right)+I_{H K} \beta_{2 n+1}$, where $\partial$ is the differentiation in $\beta_{*}$, and $I_{H K}$ is the augmentation ideal for $H K$.

Proof. Let $m \in M$. For $n \geqq 0$, denote by $A^{n}(m)$ the element of $\beta_{2 n-1}$ defined inductively by $A^{0}(m)=m, A^{n}(m)=N \otimes_{K} y \otimes_{K} A^{n-1}(m) \quad(n \geqq 1)$. (a) Since $M$ is $H$-trivial, $y A^{n}(m)=A^{n}(m)$ for $n \geqq 0$. (b) From this it follows by induction on $n$ that $\partial A^{n}(m)=0$ for $n \geqq 1$. (c) Further, since $H$ normalizes $K$ and $M$ is $K$-trivial, induction on $n$ establishes $k A^{n}(m)=A^{n}(m)$ $\forall k \in K, n \geqq 0$.

Define the $H$-homomorphism $\psi_{*}$ by

$$
\begin{aligned}
\psi_{2 n}(1 \otimes m) & =1 \otimes_{K} A^{n}(m), \\
\psi_{2 n+1}(1 \otimes m) & =1 \otimes_{K} y \otimes_{K} A^{n}(m), \quad n \geqq 0 .
\end{aligned}
$$

Then (a) implies $\partial \circ \psi_{2 n+1}=\psi_{2 n} \circ((y-1) \otimes 1)$, (b) implies $\psi_{2 n-1} \circ(N \otimes 1)=$ $\partial \circ \psi_{2 n}$ (both for $n \geqq 0$ ) so that $\psi_{*}$ is a chain map extending the identity on $M$. 
(d) Now $y \in[G, K]_{0} \cdot K$, so that $y=g k g^{-1} k_{1}^{-1}$ for some $g \in G, k, k_{1} \in K$. Thus

But

$$
\begin{array}{rlr}
y k_{1}\left(g \otimes_{K} g^{-1} \otimes_{K} A^{n}(m)\right) & =g \otimes_{K} k g^{-1} \otimes_{K} A^{n}(m) & \text { by (d), } \\
& =g \otimes_{K} g^{-1} y \otimes_{K} k_{1} A^{n}(m) & \text { by (d), } \\
& =g \otimes_{K} g^{-1} y \otimes_{K} A^{n}(m) & \text { by (c). }
\end{array}
$$

$$
\begin{aligned}
\partial\left(g \otimes_{K} g^{-1} \otimes_{K} y \otimes_{K} A^{n}(m)\right)= & \psi_{2 n+1}(1 \otimes m)-g \otimes_{K} g^{-1} y \otimes_{K} A^{n}(m) \\
& +g \otimes_{K} g^{-1} \otimes_{K} A^{n}(m) \text { by (a) and (b), }
\end{aligned}
$$

so

$$
\begin{aligned}
\psi_{2 n+1}(1 \otimes m)=\partial & \left(g \otimes_{K} g^{-1} \otimes_{K} y \otimes_{K} A^{n}(m)\right) \\
& \quad+\left(y k_{1}-1\right)\left(g \otimes_{K} g^{-1} \otimes_{K} A^{n}(m)\right), \quad n \geqq 0,
\end{aligned}
$$

which proves the lemma.

Consider the diagram

$$
\begin{gathered}
Z \otimes_{H} Y_{*} \longrightarrow Z \otimes_{H} M \\
\quad \downarrow 1 \otimes_{H} \psi_{*} \quad \downarrow \\
Z \otimes_{G} \beta_{*} \rightarrow Z \otimes_{G} M
\end{gathered}
$$

Passing to homology, the map induced by $1 \otimes_{H} \psi_{*}$ is the relative corestriction. It follows directly from the lemma that this map is zero.

Put $T=\operatorname{Ker}\left\{\beta_{2 n+1} \rightarrow \beta_{2 n}\right\}$, then we have the following commutative exact diagrams
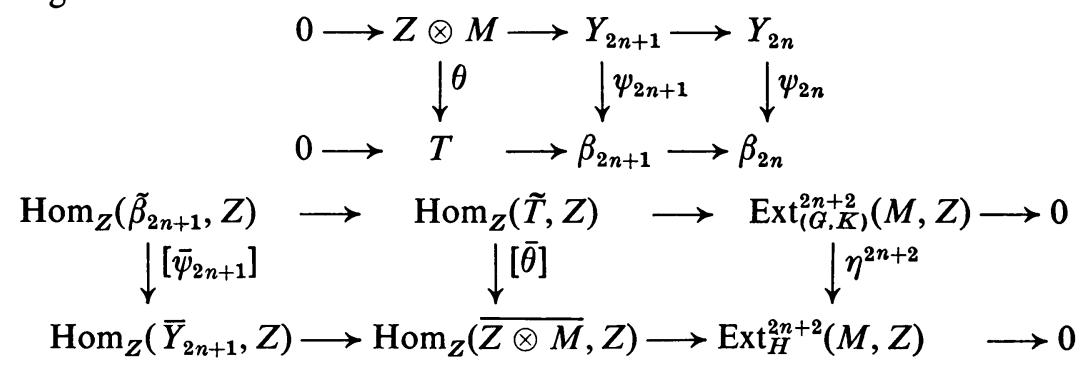

where $\tilde{X}=X \mid I_{G} X, \quad \bar{X}=X / I_{H} X$. Let $U=\operatorname{Im}\left\{\tilde{\beta}_{2 n+2} \rightarrow \tilde{\beta}_{2 n+1}\right\}$. Then $0 \rightarrow$ $H_{2 n+2}(G, K ; M) \rightarrow \tilde{T}_{\rightarrow}^{j} U \rightarrow 0$ is exact, and since $H_{*}(G, K ; M)$ has exponent $(G: K)$ [2, Chapter 28], $j$ induces an isomorphism $\phi: \operatorname{Hom}_{Z}(\widetilde{T}, Z) \cong$ $\operatorname{Hom}_{Z}(U, Z)$. From the lemma, $\bar{\psi}_{2 n+1}\left(\bar{Y}_{2 n+1}\right) \subseteq U$ and so $\left[\bar{\psi}_{2 n+1}\right] \circ \phi$ gives a map $\operatorname{Hom}_{Z}(\tilde{T}, Z) \rightarrow \operatorname{Hom}_{Z}\left(Y_{2 n+1}, Z\right)$. Since

$$
[\overline{N \otimes 1}] \circ\left[\bar{\psi}_{2 n+1}\right] \circ \phi=[\bar{\theta}],
$$

it follows that $\eta^{2 n+2}=0$, which completes the proof of the theorem. 


\section{REFERENCES}

1. I. T. Adamson, Cohomology theory for non-normal subgroups and non-normal fields, Proc. Glasgow Math. Assoc. 2 (1954), 66-76. MR 16, 442.

2. M. C. R. Butler and G. Horrocks, Classes of extensions and resolutions, Philos. Trans. Roy. Soc. London 254 (1961/62), 155-222. MR 32 \#5706.

3. L. Evens, The cohomology ring of a finite group, Trans. Amer. Math. Soc. 101 (1961), 224-239. MR 25 \#1191.

4. G. Hochschild, Relative homological algebra, Trans. Amer. Math. Soc. 82 (1956), 246-269. MR 18, 278.

5. G. Hochschild and J.-P. Serre, Cohomology of group extensions, Trans. Amer. Math. Soc. 74 (1953), 110-134. MR 14, 619.

6. S. Mac Lane, Homology, Die Grundlehren der math. Wissenschaften, Band 114, Academic Press, New York; Springer-Verlag, Berlin, 1963. MR 28 \#122.

Department of Mathematics and Statistics, University of Guelph, Guelph, Ontario, Canada 